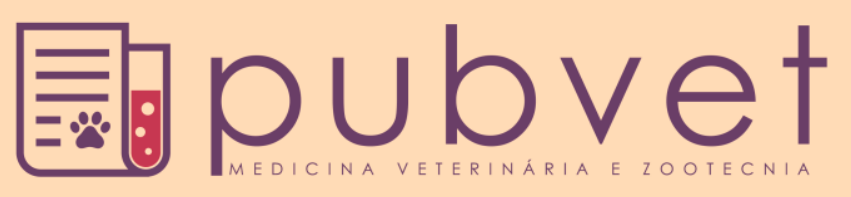

https://doi.org/10.22256/pubvet.v12n6a114.1-6

\title{
Efeitos da ioimbina, atipamezol e naloxona na reversão anestésica de macacos bugio (Aloautta guariba clamitans)
}

\author{
Sharlenne Leite da Silva Monteiro ${ }^{1 *} \bullet$, Ramiro Neves Dias Neto ${ }^{\bullet}$, Carlos Roberto \\ Teixeira $^{30}$, Gustavo Calasans Marques ${ }^{4}{ }^{\circ}$, Maíra Sales Castilho ${ }^{50}$ \\ IProfesssora da Universidade Estadual do Centro Oeste (UNICENTRO), Departamento de Medicina Veterinária, Guarapuava-PR Brasil. E- \\ mail: sharlene_monteiro@hotmail.com \\ ${ }^{2}$ Médico Veterinário do Zoológico do Rio de Janeiro (RioZoo), Rio de Janeiro-RJ Brasil. E-mail: rdiaspa@gmail.com \\ ${ }^{3}$ Professorda Faculdade de Medicina Veterinária e Zootecnia - Universidade Estadual Paulista, Departamento de Cirurgia Veterinária e \\ Anestesiologia (UNESP) Botucatu-SP Brasil.E-mail:teixeiracr@fmvz.unesp.br \\ ${ }^{4}$ Mestrando da Faculdade de Medicina Veterinária e Zootecnia - Universidade Estadual Paulista, Departamento de Cirurgia Veterinária e \\ Anestesiologia (UNESP) Botucatu-SP Brasil.E-mail: gustavocmarques@yahoo.com.br \\ ${ }^{5}$ Doutoranda da Faculdade de Medicina Veterinária e Zootecnia - Universidade Estadual Paulista, Departamento de Cirurgia Veterinária e \\ Anestesiologia (UNESP) Botucatu-SP Brasil.E-mail: maira165@gmail.com \\ *Autor para correspondência
}

RESUMO. O presente estudo abordará os efeitos causados pela contenção química com metadona $(0,1 \mathrm{mg} / \mathrm{kg})$, dexmedetomidina $(5 \mu \mathrm{g} / \mathrm{kg})$ e cetamina $(8 \mathrm{mg} / \mathrm{kg})$ em macacos Bugio (Alouatta guariba clamitans) e comparar a qualidade e o tempo na reversão anestésica com antagonistas $\alpha_{2}$-adrenérgicos isolados ou associados a antagonistas narcóticos. Foram utilizados cinco macacos bugio, adultos e hígidos, submetidos a jejum alimentar e hídrico de 12 horas. O protocolo anestésico era aplicado por via intramuscular nos músculos semimembranoso e semi-tendinoso. Cinco minutos após a sedação do animal, o mesmo era colocado em uma mesa com colchão térmico onde eram aferidos os seguintes parâmetros: frequência cardíaca, respiratória, temperatura retal, pressão arterial sistólica e reflexos palpebrais. Estes parâmetros foram aferidos em intervalos de 10 minutos. Após 40 minutos da anestesia era aplicado solução $\mathrm{NaCl} 0,9 \%$ para o tratamento controle, $25 \mu \mathrm{g} / \mathrm{kg}$ deatipamezol para o tratamento ATI, $0,5 \mathrm{mg} / \mathrm{kg}$ de ioimbina para o tratamento IOI, atipamezol $(25 \mu \mathrm{g} / \mathrm{kg})$ com naloxona $(0,2 \mathrm{mg} / \mathrm{kg})$ para o tratamento ATI NALOx $_{\text {e o }}$ tratamento IOI $_{\text {NALOX }}$ composto de ioimbina $(0,5 \mathrm{mg} / \mathrm{kg})$ associado a naloxona $(0,2 \mathrm{mg} / \mathrm{kg})$, administrados pela via intravenosa na veia ulnar dos primatas. Após a aplicação do reversor o animal era colocado em uma gaiola em decúbito dorsal, o período de recuperação total foi compreendido desde a aplicação do antagonista até a total recuperação do paciente. A recuperação foi avaliada utilizando escores variando de zero (0) a três (3). Concluiu-se que a associação metadona, dexmedetomidina e cetamina foi eficaz para contenção química desses animais permitindo a realização de procedimentos clínicos de duração de até 40 minutos. Alguns animais apresentaram sialorréia após a aplicação intravenosa de atipamezol e ioimbina. $\mathrm{O}$ tratamento $\mathrm{IOI}_{\mathrm{NALOx}}$ foi mais eficaz no quesito tempo porém, o tratamento $\mathrm{ATI}_{\mathrm{NALOX}}$ obteve a melhor recuperação.

Palavras-chave: Atipamezol, dexmedetomidina, ioimbina, primatas

\section{Effects of yohimbina, atipamezole and naloxone on the anesthetic reversal in Howley monkeys (Alaouttaguaribaclamitans)}

ABSTRACT. The present study addressed the effects caused by chemical restraint with methadone $(0,1 \mathrm{mg} / \mathrm{kg})$, dexmedetomidine $(5 \mu \mathrm{g} / \mathrm{kg})$ and ketamine $(8 \mathrm{mg} / \mathrm{kg})$ in Howley monkeys (Alouattaguaribaclamitans) and the comparison of the quality and the time of the anesthetic reversal with isolated or associated narcotic $\alpha_{2}$-adrenergic antagonists. Five adult 
and healthy Howley monkeys were submitted to food and water restraining intake of 12 hours. The anesthetic protocol was administered via intramuscular in the semimembranosus and semitendinosus muscles. Five minutes after sedation, the animal was put on a table with thermal mattress where the following parameters were measured: cardiac and respiratory frequencies, rectal temperature, systolic blood/arterial pressure and palpebral reflexes. These parameters were measured every 10 minutes. After 40 minutes of anesthesia a solution of $\mathrm{NaCl} 0.9 \%$ were administered to the control treatment, $25 \mu \mathrm{g} / \mathrm{kg}$ of atipamezole for the ATI treatment, $0,5 \mathrm{mg} / \mathrm{kg}$ of yohimbine to the IOI treatment, atipamezole $(25 \mu \mathrm{g} / \mathrm{kg})$ with naloxone $(0,2 \mathrm{mg} / \mathrm{kg})$ to the $\mathrm{ATI}_{\mathrm{NALOX}}$ treatment and the $\mathrm{IOI}_{\text {NALOX }}$ treatment was composed of yohimbine $(0,5 \mathrm{mg} / \mathrm{kg})$ associated with naloxone $(0,2$ $\mathrm{mg} / \mathrm{kg}$ ), administered via intravenous in the primates' ulnar vein. After the reverser administration the animal was placed on a cage in supine and the period of full recovery was understood from the antagonist administration until the patient's full recovery. The recovery was evaluated using scores that varied from zero (0) to three (3). The association of methadone, dexmedetomidine and ketamine was effective to the chemical restraint of these animals allowing the performance of clinical procedures during up to 40 minutes. Some animals showed drooling after the intravenous administration of atipamezole and yohimbine. The IOI $_{\text {NALOX }}$ treatment was the most effective regarding to time but the $\mathrm{ATI}_{\mathrm{NALOX}}$ treatment gained the best recovery.

Keywords: Atipamezole, dexmedetomidine, yohimbine, primate

\section{Efectos de la ioimbina, atipamezol y naloxona en la reversión anestésica de monos bugio (Aloautta guariba clamitans)}

RESUMEN. El presente estudio abordará los efectos causados por la contención química con metadona $(0,1 \mathrm{mg} / \mathrm{kg})$, dexmedetomidina $(5 \mu \mathrm{g} / \mathrm{kg})$ y cetamina $(8 \mathrm{mg} / \mathrm{kg})$ en monos Bugio (Alouatta guariba clamitans) y comparar la calidad y el tiempo en reversión anestésica con antagonistas $\alpha 2$-adrenérgicos aislados o asociados a antagonistas narcóticos. Se utilizaron cinco monos bugio, adultos e hígidos, sometidos a ayuno alimentario e hídrico de 12 horas. El protocolo anestésico se aplicó por vía intramuscular en los músculos semimembranoso y semi-tendinoso. Cinco minutos después de la sedación del animal, el mismo se colocaba en una mesa con colchón térmico donde se evaluaron los siguientes parámetros: frecuencia cardíaca, respiratoria, temperatura rectal, presión arterial sistólica y reflejos palpebrales. Estos parámetros se midieron en intervalos de 10 minutos. Después de 40 minutos de la anestesia se aplicó la solución $\mathrm{NaCl}$ 0,9\% para el tratamiento de control, 25 $\mu \mathrm{g} / \mathrm{kg}$ de atipamezol para el tratamiento ATI, $0,5 \mathrm{mg} / \mathrm{kg}$ de iohimina para el tratamiento IOI, atipamezol $(25 \mu \mathrm{g} / \mathrm{kg})$ con naloxona $(0,2 \mathrm{mg} / \mathrm{kg})$ para el tratamiento $\mathrm{ATI}_{\text {NALOx y el }}$ tratamiento $\mathrm{IOI}_{\mathrm{NALOX}}$ compuesto de ioimbina $(0,5 \mathrm{mg} / \mathrm{kg})$ asociado a naloxona $(0,2 \mathrm{mg} /$ $\mathrm{kg}$ ), administrados por vía intravenosa en la vena ulnar de los primates. Después de la aplicación del reversor el animal era colocado en una jaula en decúbito dorsal, el período de recuperación total fue comprendido desde la aplicación del antagonista hasta la total recuperación del paciente. La recuperación fue evaluada utilizando escores variando de cero (0) a tres (3). Se concluyó que la asociación metadona, dexmedetomidina y cetamina fue eficaz para contención química de esos animales permitiendo la realización de procedimientos clínicos de duración de hasta 40 minutos. Algunos animales presentaron sialorrea después de la aplicación intravenosa de atipamezol y ioimbina. El tratamiento IOI $_{\text {NALOX }}$ fue más eficaz en cuanto tiempo pero el tratamiento $\mathrm{ATI}_{\text {NALOX }}$ obtuvo la mejor recuperación.

Palabras clave:Atipamezol, dexmedetomidina, ioimbina, primates

\section{Introdução}

Para realização da maioria dos procedimentos nas espécies selvagens, faz-se necessário o emprego de contenção química, este deve garantir a realização adequada do procedimento, e principalmente, ser seguro para o animal.

Pesquisas têm sido realizadas com o intuito de se descobrir um protocolo eficaz, dotado de menor 
poder de toxicidade e que possam ser usados em animais de idade avançada, debilitados, facilmente estressáveis e, ainda, que seja de baixo custo e de fácil aquisição. Esse fármaco deve apresentar como característica desejável a facilidade de aplicação eproporcionar resultados satisfatórios na contenção e manipulação desses animais.

A contenção química deve promover a imobilização, analgesia e relaxamento muscular no animal suficiente para realização do procedimento a ser executado.

Uma recuperação anestésica de curta duração em espécies selvagens é altamente desejável. É essencial que o animal esteja perfeitamente consciente logo após sua soltura sendo capaz de se locomover, defender-se ou fugir de predadores, além de restaurar a sua hierarquia social em um grupo, evitar acidentes como quedas ou afogamentos, estresse e alterações fisiológicas, como bradipnéia e hipotermia, relacionadas ao tempo de sedação e de decúbito prolongados.

Noutro passo, observamos ainda que alguns procedimentos são relativamente curtos comparados com a duração da anestesia por isso a importância de se possuir fármacos que revertam sua ação causando uma recuperação mais rápida para o paciente.

Por fim, observamos que há uma carência na literatura do uso de protocolos anestésicos com o uso de opioides em primatas e até o presente momento, não há trabalhos com a associação metadona, dexmedetomidina e cetamina $\mathrm{S}(+)$ em Alouatta guariba clamitans, bem como, os efeitos da reversão anestésica com o uso da naloxona, ioimbina ou atipamezol nessa espécie.

Assim, esse estudo teve o objetivo de avaliar e comparar os efeitos da reversão anestésica com ioimbina $(0,2 \mathrm{mg} / \mathrm{kg})$, atipamezol $(25 \mu \mathrm{g} / \mathrm{kg})$ e naloxona $(0,2 \mathrm{mg} / \mathrm{kg}$ ) em macacos Bugio (Alouatta guariba clamitans). A hipótese seria que a associação de um antagonista $\alpha_{2}$ adrenérgico com o antagonista narcótico acelere a recuperação anestésica de forma segura e eficaz.

\section{Material e Métodos}

Este estudo foi aprovado pelo Comitê de Ética da Faculdade de Medicina Veterinária e Zootecnia (Unesp Botucatu - CEUA - 105/2014), de Meio Ambiente e Secretaria Nacional da Vida Selvagem (SISBIO - 448401), e do Instituto Chico Mendes de Conservação da Biodiversidade (ICMBio).
Foram utilizados 5 macacos Bugio (Alouatta guariaba), adultos, hígidos, pesando de $3,5-7 \mathrm{~kg}$. Os animais pertenciam ao Centro de medicina e pesquisa em animais silvestres (CEMPAS), localizado no estado de São Paulo.

Os macacos foram alimentados de acordo com as normas da instituição. Em geral, a água e uma variedade de frutas e vegetais foram fornecidas $a d$ libitum.

No dia anterior ao experimento os animais foram capturados com redes apropriadas, foi realizada a contenção física com luvas de couro. Esses ficaram em recintos individuais para a adaptação ao local do experimento, ficaram sob os cuidados dos pesquisadores onde receberam água fresca à vontade e alimentação adequada.

Foi realizado um jejum alimentar de 12 horas e hídrico de 3 horas nos animais.

Todos os animais sofreram contenção física através do uso de puçá e logo em seguida era aplicado pela via intramuscular, entre os músculos semi-tendinoso e semi-membranoso, o protocolo anestésico (metadona $\quad 0,1 \mathrm{mg} / \mathrm{kg}$, dexmedetomidina $(5 \mu \mathrm{g} / \mathrm{kg})$ com cetaminaS $(+)$ $(8 \mathrm{mg} / \mathrm{kg})$, decorrido os 5 minutos da aplicação anestésica os animais eram levados para a monitoração anestésica numa sala isolada, climatizada e sem estímulos sonoros.

Posicionado em mesa com colchão térmico eram aferidos os seguintes parâmetros: frequência cardíaca (FC) respiratória $(f)$, temperatura retal (TR) e pressão arterial sistólica (PAS) pelo método não-invasivo com a utilização de um Doppler ultrassônico com o seu sensor posicionado sobre a artéria safena e o manguito neonatal aplicado em volta do terço médio da região femoral. A temperatura foi aferida através de um termômetro digital e a FC e f foram aferidos por meio de estetoscópio. Estes parâmetros foram aferidos em intervalos de 10 minutos e devidamente anotados em fichas individuais.

Em um delineamento experimental oculto aleatorizado, os animais foram divididos em cinco grupos. Em todos os animais foi utilizado o mesmo protocolo anestésico e após 45 minutos da administração do protocolo era aplicado solução $\mathrm{NaCl} 0,9 \%$ para o tratamento controle (TC), $25 \mu \mathrm{g} / \mathrm{kg}$ de atipamezol para o tratamento ATI, 0,5 $\mathrm{mg} / \mathrm{kg}$ de ioimbina para o tratamento IOI, atipamezol $(25 \mu \mathrm{g} / \mathrm{kg})$ com naloxona $(0,2 \mathrm{mg} / \mathrm{kg})$ para o tratamento ATI NALOX e o tratamento IOI $_{\text {NALOX }}$ composto de ioimbina $(0,5 \mathrm{mg} / \mathrm{kg})$ 
associado à naloxona $(0,2 \mathrm{mg} / \mathrm{kg})$, administrados, por via intravenosa, na veia ulnar dos primatas. Após a aplicação do reversor, o animal era colocado em uma gaiola em decúbito dorsal, o período de recuperação total foi compreendido desde a aplicação do antagonista até a total recuperação do paciente, quando $o$ animal apresentava-se em pé sem ataxia.

A recuperação foi avaliada utilizando escores variando de zero (0) a três (3) de acordo com Galante (2014). Os tratamentos foram administrados por um único indivíduo não ciente do grupo estudado. Os animais foram avaliados por 3 pesquisadores não ciente do tratamento instituído.

Foi realizado o Teste de Kruskal-Wallis (não paramétrico) para comparação dos grupos $(\mathrm{P}<0,05)$. Quando significativo foi realizado o teste de Tukey para comparação das medianas.

\section{Resultados}

O score de ATI foi diferente estatisticamente de $\mathrm{ATI}_{\text {nalox }}$, porém ambos não diferiram dos demais grupos (Tabela 1).

Não houve diferença estatística entre os grupos analisados pelo avaliador 1 e pelos grupos analisados pelo avaliador 2 (Tabela 2 e Tabela 3 ).

Tabela 1. Avaliação da qualidade da recuperação anestésica pelo Score ouro.

\begin{tabular}{lc}
\hline Grupo & Mediana [percentis 25 e 75\%] \\
\hline Controle & $0,0[0,0-0,8] \mathrm{ab}$ \\
Ioimbina & $1,0[0,0-2,2] \mathrm{ab}$ \\
Atipamezol & $0,0[0,0-0,5] \mathrm{a}$ \\
Ioi+Nalox & $2,0[1,8-3,0] \mathrm{ab}$ \\
Atip+Nalox & $3,0[2,8-3,0] \mathrm{b}$ \\
\hline $\mathrm{P}$ & 0,016 \\
\hline
\end{tabular}

*Medianas seguidas pela mesma letra na coluna não diferiram estatisticamente entre si pelo Teste de Tukey $(\mathrm{P}>0,05)$.

Tabela 2. Avaliação da qualidade da recuperação anestésica pelo Avaliador 1.

\begin{tabular}{lc}
\hline Grupo & Mediana [percentis 25 e 75\%] \\
\hline Controle & $3,0[1,5-3,0] \mathrm{a}$ \\
Ioimbina & $1,0[0,0-2,2] \mathrm{a}$ \\
Atipamezol & $0,0[0,0-2,0] \mathrm{a}$ \\
Ioi+Nalox & $2,0[2,0-3,0] \mathrm{a}$ \\
Atip+Nalox & $3,0[2,5-3,0] \mathrm{a}$ \\
\hline $\mathrm{P}$ & 0,067
\end{tabular}

*Medianas seguidas pela mesma letra na coluna não diferiram estatisticamente entre si pelo Teste de Tukey $(\mathrm{P}>0,05)$.
Tabela 3. Avaliação da qualidade da recuperação anestésica pelo Avaliador 2.

\begin{tabular}{lc}
\hline Grupo & Mediana [percentis 25 e 75\%] \\
\hline Controle & $1,0[0,0-2,2] \mathrm{a}$ \\
Ioimbina & $1,0[0,0-2,0] \mathrm{a}$ \\
Atipamezol & $2,0[0,8-2,2] \mathrm{a}$ \\
Ioi+Nalox & $2,0[0,8-3,0] \mathrm{a}$ \\
Atip+Nalox & $2,0[1,8-2,0] \mathrm{a}$ \\
\hline $\mathrm{P}$ & 0,676
\end{tabular}

*Medianas seguidas pela mesma letra na coluna não diferiram estatisticamente entre si pelo Teste de Tukey $(\mathrm{P}>0,05)$.

Não houve diferença estatística entre os grupos quando comparada a mediana dos avaliadores (Tabela 4).

Tabela 4. Mediana dos avaliadores na avaliação da qualidade da recuperação anestésica.

\begin{tabular}{lc}
\hline Grupo & Mediana [percentis 25 e 75\%] \\
\hline Controle & $2,0[0,0-3,0] \mathrm{a}$ \\
Ioimbina & $1,0[0,0-2,2] \mathrm{a}$ \\
Atipamezol & $0,0[0,0-2,0] \mathrm{a}$ \\
Ioi+Nalox & $2,0[2,0-3,0] \mathrm{a}$ \\
Atip+Nalox & $3,0[1,8-3,0] \mathrm{a}$ \\
\hline $\mathrm{P}$ & 0,151
\end{tabular}

*Medianas seguidas pela mesma letra na coluna não diferiram estatisticamente entre si pelo Teste de Tukey $(\mathrm{P}>0,05)$.

Não houve diferença estatística entre os grupos para a característica tempo de recuperação (Tabela $5)$.

Tabela 5. Média \pm devio padrão do tempo em minutos da recuperação anestésica.

\begin{tabular}{lc}
\hline Grupo & Média \pm devio padrão \\
\hline Controle & $62,0 \pm 36,0 \mathrm{a}$ \\
Ioimbina & $46,6 \pm 9,5 \mathrm{a}$ \\
Atipamezol & $61,8 \pm 22,2 \mathrm{a}$ \\
Ioi+Nalox & $21,0 \pm 18,6 \mathrm{a}$ \\
Atip+Nalox & $40,2 \pm 31,0 \mathrm{a}$ \\
\hline $\mathrm{P}$ & 0,096
\end{tabular}

*Medianas seguidas pela mesma letra na coluna não diferiram estatisticamente entre si pelo Teste de Tukey $(\mathrm{P}>0,05)$.

\section{Discussão}

Dois animais apresentaram sialorréia e um apresentou nistagmo após a reversão utilizando os antagonistas $\alpha_{2}$ adrenérgicos, ioimbina e atipamezol. Reação não esperada já que a duração da cetamina é de aproximadamente 30 minutos (Ritchie et al., 1994; Harrison, 1986) e a reversão 
anestésica em nossos animais foi após 45 minutos da aplicação anestésica. Silva et al. (2010) cita em seu trabalho que após a administração da cetamina, as pupilas dilatam-se moderadamente, podendo ocorrer nistagmo, lacrimejamento e salivação, entretanto volto a salientar que já havia passado 45 minutos do efeito imediato, após aplicação, da cetamina em nosso estudo. Os opioides podem causar náuseas e vômitos (Spinosa et al., 1996). Os humanos que receberam morfina e pequenas doses de cetamina apresentaram na recuperação anestésica náuseas e vômitos (Winterborn et al., 2008). Fato que pode ter ocorrido em nossa pesquisa, ao antagonizar o agonista $\alpha_{2}$ adrenérgico restaram o opioide associado a cetamina essa associação pode ter prologando os efeitos adversos nesses animais.

Estatisticamente não houve diferenças significativas quanto ao tempo de recuperação entre os tratamentos. No tratamento ATI a média da recuperação foi de $61,8 \pm 22,2$ min diferente do encontrado por Vié et al (1998) que obteve uma média de 51,0 $\pm 23,2$ min ao antagonizar os efeitos da medetomidina $150 \mu \mathrm{g} / \mathrm{kg}$ associada a cetamina $4 \mathrm{mg} / \mathrm{kg}$ com atipamezol (763 \pm 38 $\mu \mathrm{g} / \mathrm{kg}$ ) em Bugios ruivos (Alouatta seniculus) mas o tratamento $\mathrm{ATI}_{\text {nalox }}$ teve uma recuperação de $40,2 \pm 31 \mathrm{~min}$. Isso demonstra que no tratamento ATI a recuperação foi prolongada devido o efeito residual da metadona associada a cetamina $\mathrm{S}(+)$ e ao se antagonizar os dois fármacos houve uma diminuição do tempo de recuperação. $\mathrm{O}$ mesmo ocorreu com o tratamento IOI onde a média de recuperação foi de 46,6 \pm 9,5 minutos e o tratamento $\mathrm{IOI}_{\text {nalox }}$ que foi de $21 \pm 18,6$ minutos. Kreeger \& Seal (1986) consideraram que a ioimbina exerce um efeito antagonista parcial sobre a cetamina, bloqueando alguns dos subtipos de receptores, mas não antagoniza seus efeitos totais isso poderia justificar o tempo de recuperação dos tratamentos IOI e IOI $_{\text {nalox }}$ do nosso trabalho ter sido menor que os tratamentos ATI e $\mathrm{ATI}_{\text {nalox }}$, porém mais estudos seriam necessários para avaliar esse fenômeno.

A ioimbina $(0,3 \mathrm{mg} / \mathrm{kg})$ antagonizou significativamente melhor que o atipamezol $(0,1 \mathrm{mg} / \mathrm{kg})$ a hipotermia causada pela aplicação de medetomidina $(0,3 \mu \mathrm{g} / \mathrm{kg})$ em ratos reduzindo os seus efeitos farmacológicos e o tempo de recuperação (Haapalinna et al., 1997).

A relação de seletividade $\alpha_{2}: \alpha_{1}$ da medetomidina em ratos é de 1600:1 e a seletividade do atipamezol e ioimbina seria
8526:1, 40:1, respectivamente (Bacchiega \& Simas, 2008; Talukder et al., 2009).

Observamos que a seletividade do atipamezol aos receptores $\alpha_{2}$ é 200 a 300 vezes superior à ioimbina. A reversão dos efeitos da medetomidina pelo atipamezol em cães é obtida com a administração de doses 4 a 6 vezes superiores às do agonista. Em felinos as doses necessárias são 2 a 4 vezes maiores (Cullen, 1996). A dose usada em nosso estudo foi de $25 \mu \mathrm{g} / \mathrm{kg}$ de atipamezol que seria 5 vezes a dose da dexmedetomidina (5 $\mu \mathrm{g} / \mathrm{kg}$ ) e a da ioimbina foi de $0,2 \mathrm{mg} / \mathrm{kg}$ que seria 40 vezes a dose do agonista $\alpha_{2}$ adrenérgico.

A naloxona é desprovida de atividade intrínseca, mas possui mesma afinidade pelos receptores opioides. Ao se ligar ao receptor, a molécula da naloxona ocupa o receptor deslocando o agonista opioide de seu sítio, impedido o aparecimento de seu efeito, sem ativar os mecanismos celulares que decorrem da ligação de um agonista opioide nesse receptor.

Três pesquisadores avaliaram a qualidade da recuperação anestésica dos animais e entre eles não houve diferenças significativas nos tratamentos. Mas houve uma discordância entre os avaliadores, na avaliação do Score ouro (que é representado por pesquisador mais experiente nesse tipo de avaliação) houve diferença significativa entre os tratamentos ATI e $\mathrm{ATI}_{\text {nalox }}$ com os demais tratamentos. O tratamento ATI obteve a pior recuperação, o animal tem retorno agitado, com severa ataxia e risco de injúria e o grupo $\mathrm{ATI}_{\text {naloxa }}$ melhor recuperação, $\mathrm{o}$ animal apresenta mudança de decúbito com mínimos movimentos atáxicos, se levanta com uma ou duas tentativas e é capaz de ambular com somete ligeira ataxia. Os pesquisadores 1 e 2 não obtiveram diferenças significativas nas suas avaliações havendo uma concordância entre eles.

\section{Referências bibliográficas}

Bacchiega, T. S. \& Simas R. C. 2008. Dexmedetomidina um novo medicamento na anestesiologia veterinária. Revista Científica Eletrônica de Medicina Veterinária,Ano VI (10).

Cullen, L. K. 1996. Medetomidine sedation in dogs and cats: a review of its pharmacology, antagonism and dose. The British Veterinary Journal, 152(5), 519-535.

Galante, R., Muniz, J. A., Castro, P. H., Gris, V. N., Carvalho, E. R., Amora, D. S. \& Vilani, R. 
G. 2014 Continuous infusion of propofol or intermittent bolus of tiletamine-zolazepam in squirrel monkeys (Saimiri sciureus). Veterinary Anaesthesia and Analgesia, 41(5), 506-515.

Haapalinna, A., Viitamaa, T., MacDonald, E., Savola, J. M., Tuomisto, L., Virtanen, R. \& Heinonen, E. 1997, Evaluation of the effects of a specific alpha 2-adrenoceptor antagonist, atipamezole, on alpha 1- and alpha 2adrenoceptor subtype binding, brain neurochemistry and behaviour in comparison with yohimbine. Naunyn Schmiedebergs Archives Pharmacology, 356(5), 570-582.

Harrison G. J. 1996, Anesthesiology. In: Harrison G. J. \& Harrison, L. R. (eds.). Clinical Avian. Medicine and Surgery (P. 549-559). WB Saunders, Philadelphia, US.

Kreeger, T. J. \& Seal, U. S. 1986. Failure of yohimbine hydrochloride to antagonize ketamine hydrochloride immobilization of gray wolves. Journal of Wildlife Diseases, 22(4), 600-603.

Ritchie, B. W., Harrison, G. J. \& Harrison, L. R. 1994. Avian medicine: principes and aplication. Wingers Publishing, Florida, US.

Silva, F. C. C., Dantas, R. T., Citó, M. C., Silva, M. I., Vasconcelos, S. M. \& Fonteles, M. M. 2010. Ketamina, da anestesia ao uso abusivo: artigo de revisão. Revista Neurociência, 18(2), 227-237.
Spinosa, H. S., Gorniak, S. L. \& Bernardi, M. M. 1996. Farmacologia aplicada a medicina veterinária. Guanabara Koogan, Rio de Janeiro, BR.

Talukder, H., Hikasa, Y., Takahashi, H., Sato, K. \& Matsuu, A. 2009. Antagonistic effects of atipamezole and yohimbine on medetomidineinduced diuresis in healthy dogs. The Canadian Journal of Veterinary Research, 73(4), 260-270.

Vié, J. C., De Thousy, B., Fournier, P., FournierChambrillon, C., Genty, C. \& Kéravec, J. 1998. Anesthesia of wild red howler monkeys (Alouatta seniculus) with medetomidine/ketamine and reversal by atipamezole. American Journal of Primatology,45(4), 399-410.

Winterborn, A. N., Bates, W. A., Feng, C. \& Wyatt, J. D. 2008. The efficacy of orally dosed ketamine and ketamine/medetomidine compared with intramuscular ketamine in rhesus macaques (Macaca mulatta) and the effects of dosing route on haematological stress markers. Journal Medical of Primatology, 37(3), 116127.

Article History:

Received 7 March 2018

Accepted 26 March 2018

Available online 31 May 2018

License information: This is an open-access article distributed under the terms of the Creative Commons Attribution License 4.0, which permits unrestricted use, distribution, and reproduction in any medium, provided the original work is properly cited. 